\title{
Dynamical evolution of the inner heliosphere approaching solar activity maximum: interpreting Ulysses observations using a global MHD model
}

\author{
P. Riley, Z. Mikić, and J. A. Linker \\ Science Applications International Corporation, 10260 Campus Point Dr., San Diego, CA 92121, USA
}

Received: 31 October 2002 - Revised: 2 February 2003 - Accepted: 7 February 2003

\begin{abstract}
In this study we describe a series of MHD simulations covering the time period from 12 January 1999 to 19 September 2001 (Carrington Rotation 1945 to 1980). This interval coincided with: (1) the Sun's approach toward solar maximum; and (2) Ulysses' second descent to the southern polar regions, rapid latitude scan, and arrival into the northern polar regions. We focus on the evolution of several key parameters during this time, including the photospheric magnetic field, the computed coronal hole boundaries, the computed velocity profile near the Sun, and the plasma and magnetic field parameters at the location of Ulysses. The model results provide a global context for interpreting the often complex in situ measurements. We also present a heuristic explanation of stream dynamics to describe the morphology of interaction regions at solar maximum and contrast it with the picture that resulted from Ulysses' first orbit, which occurred during more quiescent solar conditions. The simulation results described here are available at: http://sun.saic.com.
\end{abstract}

Key words. Interplanetary physics (Interplanetary magnetic fields; solar wind plasma; sources of the solar wind)

\section{Introduction}

Beyond $\sim 10$ solar radii $\left(R_{S}\right)$, solar material streams away from the Sun along roughly radial trajectories. A combination of temporal variations at the Sun, together with the rotation of the Sun, leads to parcels of plasma with different plasma and magnetic properties becoming radially aligned: faster material overtaking slower material leads to a compression front, while slower material being outrun by faster material leads to a rarefaction region, or expansion wave (Sarabhai, 1963). When the flow pattern at the Sun does not vary considerably during a solar rotation (such as during solar minimum), the large-scale compressive structures created by the interaction of these streams are fixed in a frame coro-

Correspondence to: P. Riley (pete.riley@ saic.com) tating with the Sun, and they are known as corotating interaction regions (CIRs) (Smith and Wolfe, 1976). When the difference in speed between the slow and fast streams is sufficiently large, a pair of shocks may form bounding the CIR (Pizzo, 1985).

During its initial voyage out of the ecliptic plane, Ulysses observed a systematic orientation of the CIRs it encountered (Gosling et al., 1993, 1995; Riley et al., 1996; GonzalezEsparza and Smith, 1997). These interaction regions were observed from January 1992 to October 1993, corresponding to the declining phase of solar cycle 22. In the azimuthal $(\phi)$ plane their outward normals were tilted westward (i.e. into the direction of planetary motion). This was to be expected, as the interaction regions aligned themselves roughly along the direction of the Parker spiral. In the meridional plane $(\theta)$ their outward normals were tilted toward the heliographic equator. These results were supported by numerical MHD simulations (Pizzo, 1994) which suggested that these orientations were a natural consequence of the tilt of the solar magnetic dipole axis relative to the solar rotation axis. This pattern of tilts was once again observed during Ulysses' socalled "rapid latitude scan" (Gosling et al., 1995) and, most recently, as Ulysses descended from the northen polar regions to the equator (Gosling et al, 1997; Mc Comas et al., 1998).

Previous solar wind models have yielded considerable insight into the dynamical processes that shape the structure of the heliosphere. The large-scale, time-stationary structure of the heliosphere under an idealized tilted-dipole geometry has been studied in detail by V. J. Pizzo (e.g. Pizzo, 1991; Pizzo and Gosling, 1994, and references therein). At an inner boundary of $32 R_{S}$ a flow pattern was constructed consisting of a slow, dense flow about a heliomagnetic equator that was tilted by a specified amount relative to the rotational axis. Similar simulations were used by Pizzo (1994) to interpret Ulysses observations at mid-heliographic latitudes, where it was found that CIR-associated reverse shocks persist to much higher latitudes than CIR-associated forward shocks. These results suggested that the tilted-dipole geom- 
etry at the inner boundary strongly drives the position and orientation of the CIRs and their associated shocks. In particular, under moderately low solar activity conditions, CIRs and shock outward normals are tilted toward the equator in both hemispheres. Thus, in the ecliptic plane a spacecraft samples alternating tilts as it passes through a predominantly Northern Hemispheric CIR and, subsequently, a predominantly Southern Hemispheric CIR. Moreover, the strongest interactions take place away from the ecliptic plane, at latitudes roughly equal to the tilt of the solar dipole relative to the rotation axis.

Wang and Sheeley (1990) exploited an empirical relationship between solar wind speed and coronal flux tube expansion (Levine et al., 1977) to predict solar wind speed at $1 \mathrm{AU}$ and beyond. They found that coronal flux tubes that expand more slowly correlate with faster asymptotic speed along that flux tube. Using this relationship, they predicted the types of wind speed patterns that Ulysses would be expected to see during its second solar orbit (Wang and Sheeley, 1997). In particular, they predicted that the ascending phase of the solar cycle would be dominated by recurrent (28-29 day periodicity), high-speed streams originating from high-latitude extensions of polar coronal holes. Approaching solar maximum, however, persistent high-speed streams would disappear, only to be replaced by low-speed wind at all latitudes. Finally, at solar maximum (or, more specifically, at the time that the polar field reverses), very fast episodic polar jets would be generated as active region fields migrated toward the solar poles.

The data utilized in this study derive from the Solar Wind Observations Over the Poles of the Sun (SWOOPS) ion sensor (Bame et al., 1992) and the magnetic field investigation (Balogh, 1992) on board the Ulysses spacecraft. The plasma moments have a typical resolution of 4-8 min while the magnetic field measurements have a resolution of 1-2 s. All data presented here, however, have been averaged over 1 hour.

The purpose of this report is twofold. First, we describe a series of MHD simulations covering approximately 3 years during the late ascending phase of the solar cycle and through solar maximum and use them to interpret Ulysses in situ observations. Second, we present a simple explanation for both the observed and modeled stream structure at solar maximum, and contrast it with solar minimum conditions. The report is structured as follows. In Sect. 2 we describe the basic features and approximations inherent to the model. In Sect. 3 was discuss the properties and evolution of the solar wind as seen by Ulysses during the time period from 12 January 1999 to 19 September 2001. In Sect. 4 we describe the global evolution of the inner heliosphere as inferred from the MHD simulations for the same time period. We explore two specific intervals in more detail in Sect. 5 to highlight the differences in stream structure between solar minimum and maximum. Finally, in Sect. 6 we summarize the main points of the study, address some of the limitations and sources of errors in the simulations, and discuss possible areas for future studies.

\section{Description of the model}

We use a three-dimensional, time-dependent MHD model, driven by the observed line-of-sight photospheric magnetic field to model the structure of the inner heliosphere $\left(1 R_{S}\right.$ to $5 \mathrm{AU})$. The details of the algorithm have been discussed by Mikić et al. (1999) and Linker et al. (1999) and its extension from the solar corona to the inner heliosphere is discussed by Riley et al. (2001a,b). Briefly speaking, we separate the region of space from $1 R_{S}$ to $5 \mathrm{AU}$ into two distinct regions: the coronal model, which spans $1 R_{S}$ to $30 R_{S}$ and the heliospheric model, which spans $30 R_{S}$ to $5 \mathrm{AU}$. Such an approach is both computationally more efficient and produces a more realistic heliospheric environment.

The heliospheric boundary conditions are specified in the following way:

1. The radial component of the coronal magnetic field at $30 R_{S}$ is used directly as the inner boundary condition for $B_{r}$.

2. The speed is computed using the coronal magnetic configuration: at $1 R_{S}$ we set the radial speed to be some value, $v_{\text {slow }}$, at the boundary between open and closed field lines over a width of $\sim 6^{\circ}$ (in a direction normal to the boundary) and smoothly raise it to $v_{\text {fast }}$ over $\sim 3^{\circ}$. We then map this speed profile outward along the open field lines to $30 R_{S}$. Although this may appear somewhat ad hoc, it is based on the commonly held view that fast wind emanates from within coronal holes and slow wind is associated with the boundary between open and closed fields, as would be the case if closed field lines were sporadically opened, through magnetic reconnection. Such an approach is required because the polytropic approximation used in the coronal solution does not yield sufficiently large variations between the slow and fast wind.

3. We assume a momentum flux balance at the inner boundary, which specifies the plasma density.

4. We assume thermal pressure balance, yielding the plasma temperature. Comparisons with in situ observations suggest that this approach is capable of capturing the essential features of the large-scale structure of the inner heliosphere for a variety of solar conditions (Riley et al., 2001a,b).

Riley et al. (2001a) have discussed the approximations of this model in detail. Here we make a few brief remarks. First, we neglect the effect of pickup ions, which are thought to dominate the internal energy of the solar wind beyond 6$10 \mathrm{AU}$ (Axford, 1973). Thus, we limit our modeling region to $\sim 5 \mathrm{AU}$. Second, we neglect the effects of differential rotation, which may play a role in connecting high-latitude field lines near the Sun with lower-latitude interaction regions much further away (Fisk, 1996). Third, although our MHD model is time-dependent, we assume that the flow at the inner boundary is time-stationary. Thus, the flow at the inner 
boundary rotates rigidly with a period of 25.38 days and spatial variations are responsible for the generation of dynamic phenomena in the solution. Consequently, our results do not include transient phenomena, such as coronal mass ejections. Finally, the grid resolution necessary to model a region of space spanning $5 \mathrm{AU}$ in radius precludes us from accurately modeling shock waves.

Since the model, as implemented here, is driven by synoptic maps of the line-of-sight photospheric magnetic field observed at the Kitt Peak observatory, each solution describes an "average" picture for that Carrington rotation. And, while the solution does not contain any transient phenomena, it can be interpreted as the "underlying" solution, assuming that the magnetic configuration responsible for the transient event returned to its initial state after eruption. In spite of these limitations, even under solar maximum conditions, this approach can produce results that compare favorably with in situ observations (Riley et al., 2002).

\section{Ulysses observations}

By early 1999, the Ulysses spacecraft had already begun its second excursion from the ecliptic plane toward the sourthern polar regions of the Sun. Moving in from 5.19 AU and $-19.6^{\circ}$ heliographic latitude on 12 January 1999 , Ulysses reached a peak southern latitude of $-80.2^{\circ}$ on 23 November 2000 (day 328) and then swung rapidly from the southern polar regions to the northern polar regions, crossing the equator on 17 May 2001 (day 137) at a distance of $1.34 \mathrm{AU}$ and reaching a peak northern latitude of $+80.2^{\circ}$ on 11 October 2001 (day 284). In this study, we focus on CR1945CR1980, corresponding to the time period 12 January 1999 to 19 September 2001. Figure 1 summarizes Ulysses' trajectory during this time period (bottom panel), together with speed, proton number density ( $n_{p}$, scaled by $r^{2}$ to account for the spherical expansion of the solar wind) and proton temperature ( $T_{p}$, scaled by $r$ to account for the near-adiabatic expansion of the solar wind). We see that the majority of solar wind intercepted by Ulysses was relatively slow and variable. In fact, in 1999 and 2000, the wind only exceeded $600 \mathrm{~km} \mathrm{~s}^{-} 1$ a few times. By the time Ulysses had reached $\sim 60^{\circ} \mathrm{N}$ heliolatitude, substantial intervals of high-speed wind were once again observed (McComas et al., 2002b). A clear transition occurred in August 2001, however, when the spacecraft became immersed in high-speed, tenuous, and quescient solar wind, similar to what was observed over both poles during its first orbit. At this point, Ulysses had crossed the solar pole and was back at $\sim 60^{\circ} \mathrm{N}$ heliolatitude. Also of note is that the slowest density and coldest flow occurred during late 2000 and early 2001 when the average speed remained less than $400 \mathrm{~km} \mathrm{~s}^{-1}$. The structure of the solar wind, as inferred from these plasma measurements, has been discussed in detail by McComas et al. (2000, 2002a,b); McComas (2002).
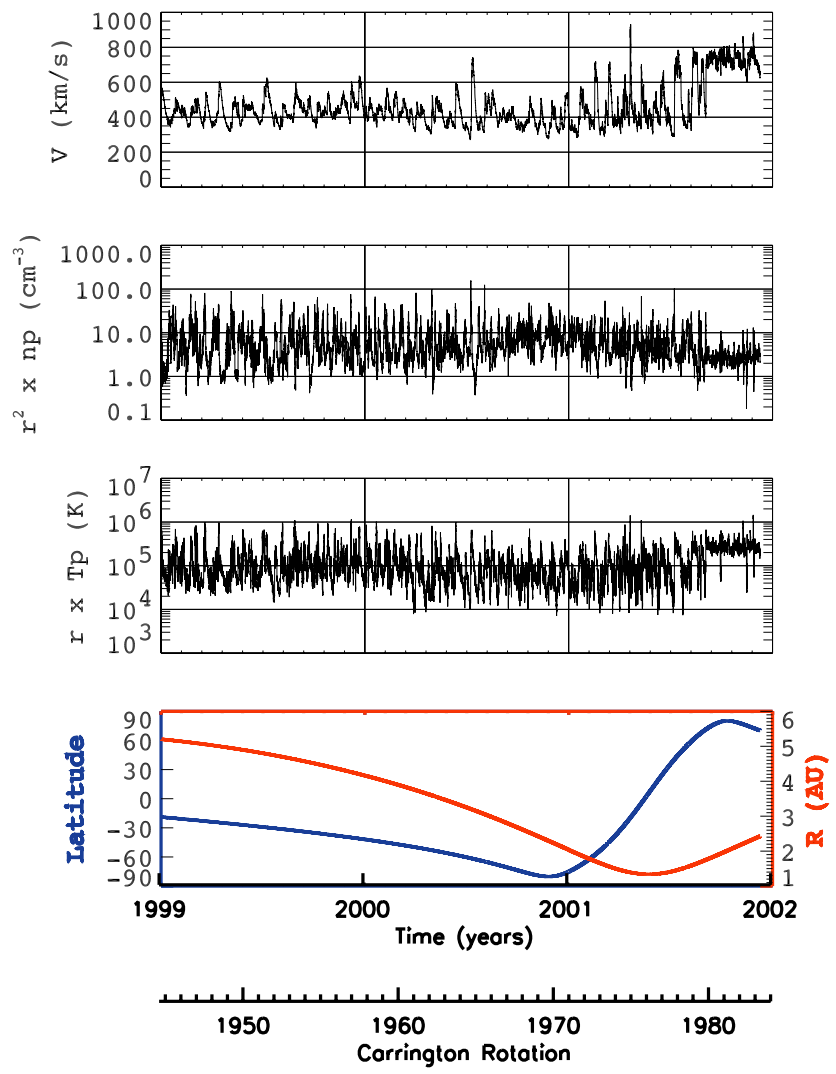

Fig. 1. Ulysses measurements (1-h averages) of solar wind speed, density, and temperature, together with the spacecraft's location (radial distance: red, and heliographic latitude: blue) from 12 January 1999 to 19 September 2001.

\section{Evolution of the solar wind approaching solar maxi- mum}

The inputs used to construct the model solutions for CR1945 to CR1980 are shown in Fig. 2, where Carrington longitude $\left(0^{\circ}-360^{\circ}\right)$ runs along the $\mathrm{x}$-axis and heliographic latitude $\left(-90^{\circ}\right.$ to $\left.+90^{\circ}\right)$ runs along the $y$-axis. These were derived from Kitt Peak measurements of the line-of-sight photospheric magnetic field, and have undergone significant processing to provide more appropriate boundary conditions for the simulations. Since these inputs are the principal driver of the model, it is worth discussing this method in some detail. First, accurate measurements of the polar fields (say above $\pm 70^{\circ}$ ) are difficult because of line-of-sight projection effects. Thus, rather than use unreliable data, measurements within $20^{\circ}$ of each pole were replaced by values extrapolated from a two-term Taylor series fit of the axisymmetric component of the observed field between $14^{\circ}$ and $28^{\circ}$ of the poles. Second, any small residual monopolar component to the field was subtracted out. Third, the data were interpolated to the resolution used in the simulation, while preserving the magnetic flux in the interpolation. Fourth, a longitudinal filter of the form $e^{-\left(0.7 m / m_{\max }\right)^{8}}$ was applied, where $m_{\max }$ specifies the mode that is attenuated to $95 \%$ of its original amplitude, 


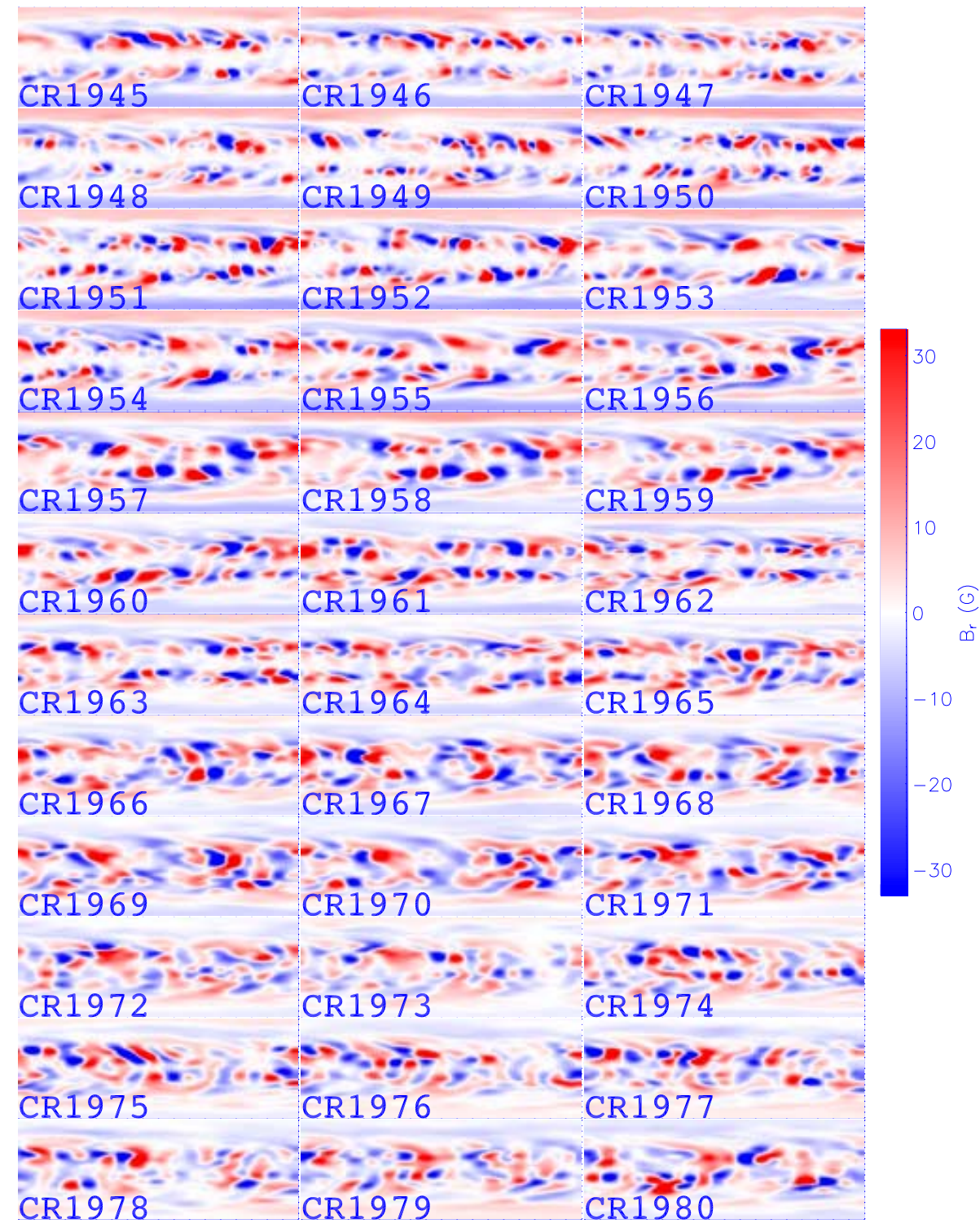

Fig. 2. Radial magnetic field at the photosphere for CR1945 to CR1980, derived from Kitt Peak synoptic maps.

and modes with $m$ smaller than $m_{\max }$ are not attenuated appreciably. For these simulations, $m_{\max }=9$ was chosen. The resulting values provide the radial component of the magnetic field at our inner boundary, $B_{r 0}$. We caution that a comparison of these results with the original Kitt Peak magnetograms reveal some significant differences (Linker et al., 1999). In particular, the small-scale "peppered" appearance is replaced with large-scale bipole regions, preferentially selected by the particular value of $m_{\max }$ chosen. On the other hand, the large-scale structure of the data is well preserved.

Scanning the panels in Fig. 2, we note that, in general, there is significant evolution from one rotation to the next. However, many features persist for several rotations. The most striking trend, however, is the reversal of the polar fields during this time span. Initially, the polar fields are outward (red) in the Northern Hemisphere and inward (blue) in the Southern Hemisphere. By the end of the time period they are outward in the Southern Hemisphere and inward in the Northern Hemisphere. The mid-point likely occurred between CR1969 and CR1973, or 27 October 2000-13 March 2001 (inferred by saturating the colors in Fig. 2).

The specification of the remaining boundary conditions, as well as the initial condition for the magnetofluid variables, has been described by Linker et al. (1999) and are not repeated here. Given this initial state and boundary conditions the MHD equations are then integrated forward in time until a steady state equilibrium is achieved. This procedure was repeated for each Carrington rotation until a database of 36 coronal solutions was obtained.

These solutions allow us to compute coronal hole boundaries, i.e. the boundary between open and closed magnetic field lines, by tracing along magnetic field lines and determining whether they are open (i.e. extend out through the outer boundary of the coronal solution) or are closed (i.e. re- 


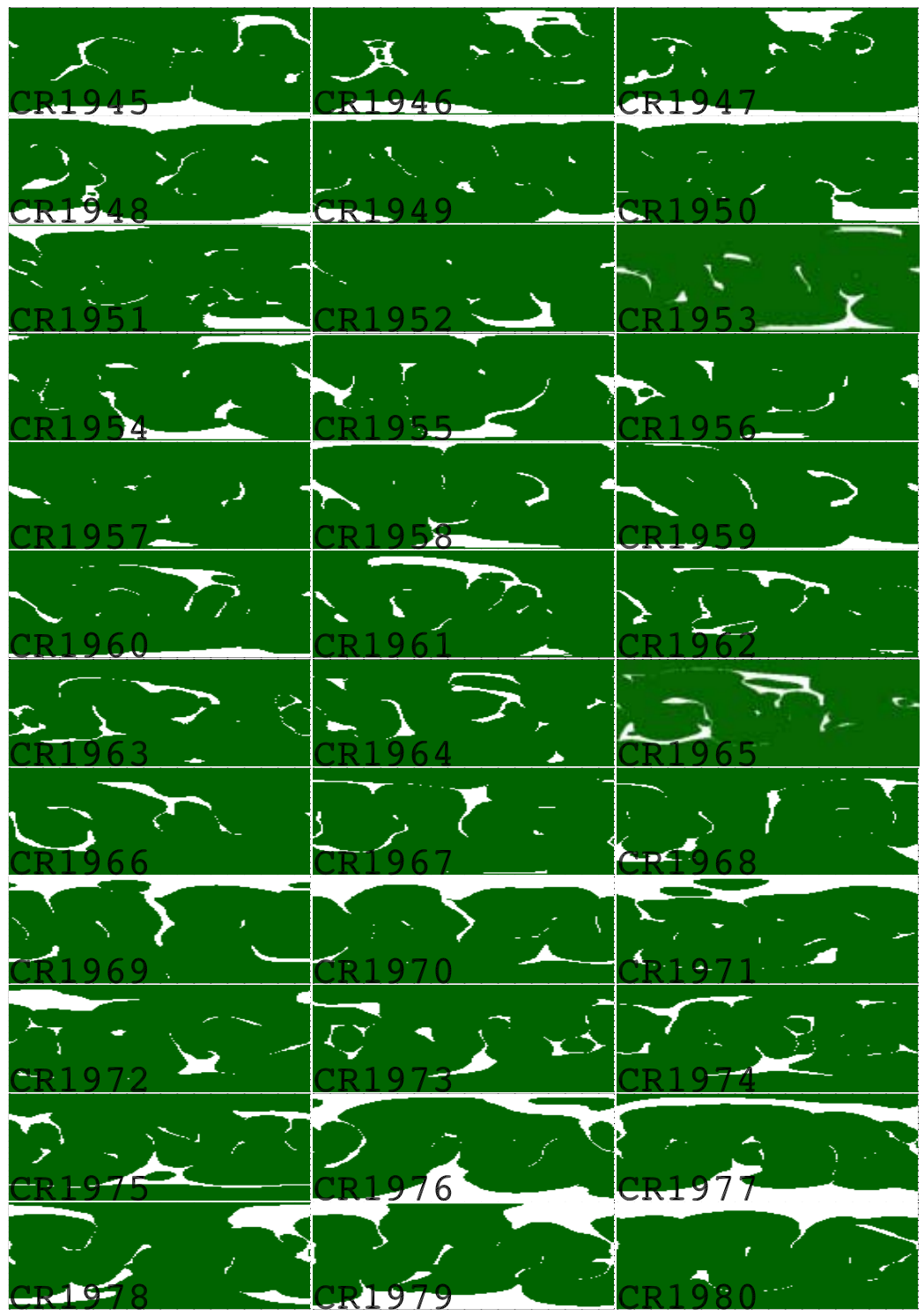

Fig. 3. Computed coronal hole boundaries for CR1945 to CR1980. Green indicates regions of closed magnetic fields and white indicates regions of open magnetic fields (i.e. coronal holes).

turn back to the surface of the Sun). We emphasize that these computed boundaries are not necessarily the same as coronal hole boundaries computed from Kitt Peak He I $1083 \mathrm{~nm}$ observations. In fact, they are typically a superset. This may signify an error in the modeling, or may suggest that the observations do not always identify the boundaries well. We should also mention that there is an implicit, although reasonable assumption that coronal holes are in fact defined by open field lines.

In Fig. 3 we summarize the computed coronal hole boundaries. The large-scale polar coronal holes that were consistently present during Ulysses' first orbit are gone, replaced instead by smaller coronal holes located at essentially all heliographic latitudes. Initially, a small southern coronal hole is present for 4 rotations or so. We should be cautious, however, about over-interpreting results at the highest heliolatitudes, since our accuracy there is the poorest. A fairly substantial northern coronal hole was present in rotations 1969-1971, and there is an indication that polar coronal holes are again returning toward the end of the time period. The overwhelming feature of these maps, however, is how small the fraction of the solar surface is that is covered by coronal holes. Typically, they account for only $\sim 6 \%$. Comparison of Fig. 3 with Fig. 2 highlights the importance of MHD (or potential field source surface) models in revealing the magnetic field structure of the corona. It would be difficult, if not impossible, to infer the topology of magnetic field lines and/or the location or coronal holes based only on the photospheric field data. A detailed comparison of these results with Kitt Peak He I $1083 \mathrm{~nm}$ observations, while clearly of importance, is beyond the scope of the present study.

As discussed above, and in more detail by Riley et al. 


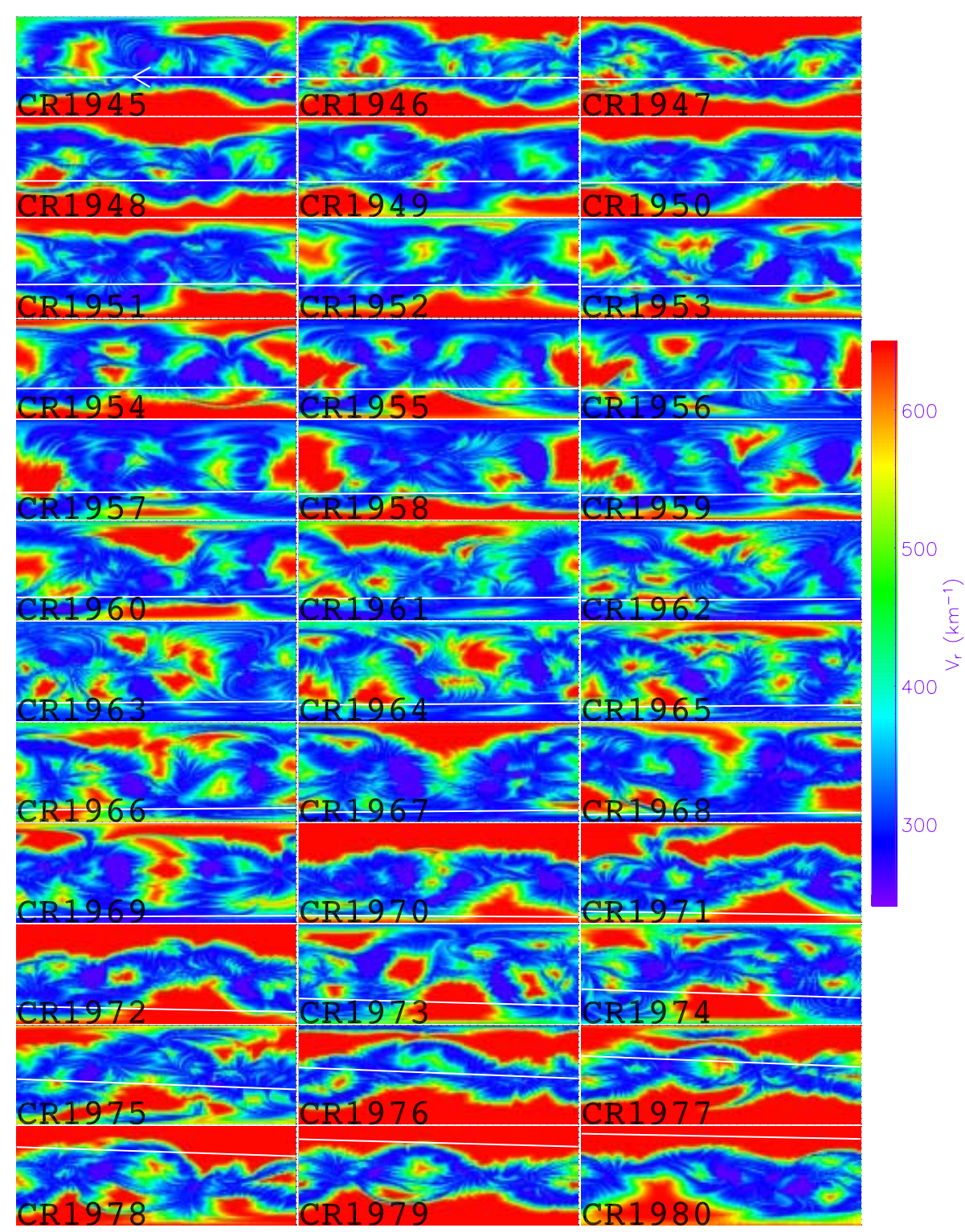

Fig. 4. Computed radial velocities for CR1945 to CR1980. The solid line in each panel indicates Ulysses' mapped trajectory along this spherical shell.

(2001a,b), the coronal solutions are used to calculate the boundary conditions for the heliospheric portion of the model at $30 R_{S}$. The radial component of the magnetic field is used directly, while the velocity, density, and temperature are calculated in a somewhat more ad hoc way. The computed speeds at $30 R_{S}$ are shown in Fig. 4. Ulysses' trajectory has also been projected back to the Sun for each Carrington rotation and is displayed as a white line. Time runs in the opposite sense to longitude, and so it increases from right to left.

The flow speed of the solar wind at $30 R_{S}$ is not typically considered to be a fundamental parameter, since measurements in this region are difficult to make and hence sparse. Nevertheless, they are an excellent tool for interpreting the global context of in situ measurements. At this distance, all super-radial expansion has occurred and the flow is essentially radial, yet no stream dynamics have taken place to any significant extent, complicating the flow pattern. Thus, we have the potential to resolve such questions as to whether the presence of an intermediate-speed stream (say, $600 \mathrm{~km} \mathrm{~s}^{-1}$ ) was the result of the spacecraft grazing the edge of a substantial coronal hole, or intercepting a smaller high-speed stream that has decelerated, via the interaction with slow solar wind during its passage to the spacecraft (stream attenuation). We are currently studying these simulations in greater detail to assess how well the computed stream structure matches observations at Ulysses, WIND, and ACE. Previously (Riley et al., 2001a,b), we have shown that they can reproduce the essential stream structure patterns, particularly at solar minimum. We realize, however, that at solar maximum, temporal 
variations at the Sun will undoubtedly lead to streams that cannot be modeled with our synoptic solutions and we do not expect to match the observations all of the time.

There is a clear evolutionary trend to the stream structure shown in Fig. 4. Initially, a pair of high speed streams emanate from both poles. By CR1952 the northern high-speed stream has disappeared and by CR1959, the southern high speed stream is gone. Ulysses at this time was traveling from low latitudes toward the southern polar regions, and, as can be seen in Fig. 4, chased the receding coronal hole without ever becoming immersed within in. Between CR1959 and CR1968 only smaller high-speed streams were present. Ulysses intercepted them, but by the time they had pushed their way out to the location of the spacecraft, they were reduced to a speed of less than $600 \mathrm{~km} \mathrm{~s}^{-1}$. During rotations CR1969 - CR1972 a fairly substantial high-speed stream was present over the northern polar region, but Ulysses, still being in the Southern Hemisphere, had no direct knowledge of it. However, by the time Ulysses had reached equatorial regions during its rapid latitude scan, both the northern and southern regions were developing polar coronal holes and hence high-speed flow. Ulysses intercepted the southern edge of the northern polar stream in CR1978, and became completely immersed in it the following rotation.

Although we have emphasized the high-speed streams in our discussion, we could have equally well described Ulysses' journey relative to the slower, more variable wind. During a few rotations, such as CR1947 and CR1976, we see the "tilted-dipole" picture, as suggested by the idealized streams modeled by Pizzo (1994), where the slow wind is confined to a constant-width, sinusoidally-varying profile with respect to longitude. More often, we see the solarmaximum picture of isolated high-speed streams, with slow flow everywhere else. In addition, even when the polar coronal holes have returned, the structure of the slow-flow band is significantly different from the "tilted-dipole" picture. In particular, the band does not have a constant width. More often it resembles a set of sausage links, and rather than having a sinusoidal variation, it is flat, with localized warps. These differences are likely due to the contribution of the quadrupole and octupole components to the magnetic field.

As with the comparison of Figs. 2 and 3, there is not a clear correspondence between patterns in the coronal holes (Fig. 3) and the inferred radial speed (Fig. 4). In most cases we can identify the coronal hole that is responsible for a particular high-speed stream, yet the morphology of the streams is not predictable from the coronal hole structure, because it derives from the full structure of the coronal magnetic field.

The evolution of the average properties of the parameters shown in Figs. 2-4 are shown in Fig. 5. In the top panel, the average value of the absolute value of $B_{r}$ is shown. And, although it does not show any obvious trend with solar cycle, there is significant variation during the 36 Carrington rotations; from a minimum of 2.4 to a maximum of 4 , a change of $\sim 67 \%$. The middle panel shows the fractional area covered by coronal holes, obtained by integrating over the solar surface, and accounting for the sin(latitude) decrease in el-
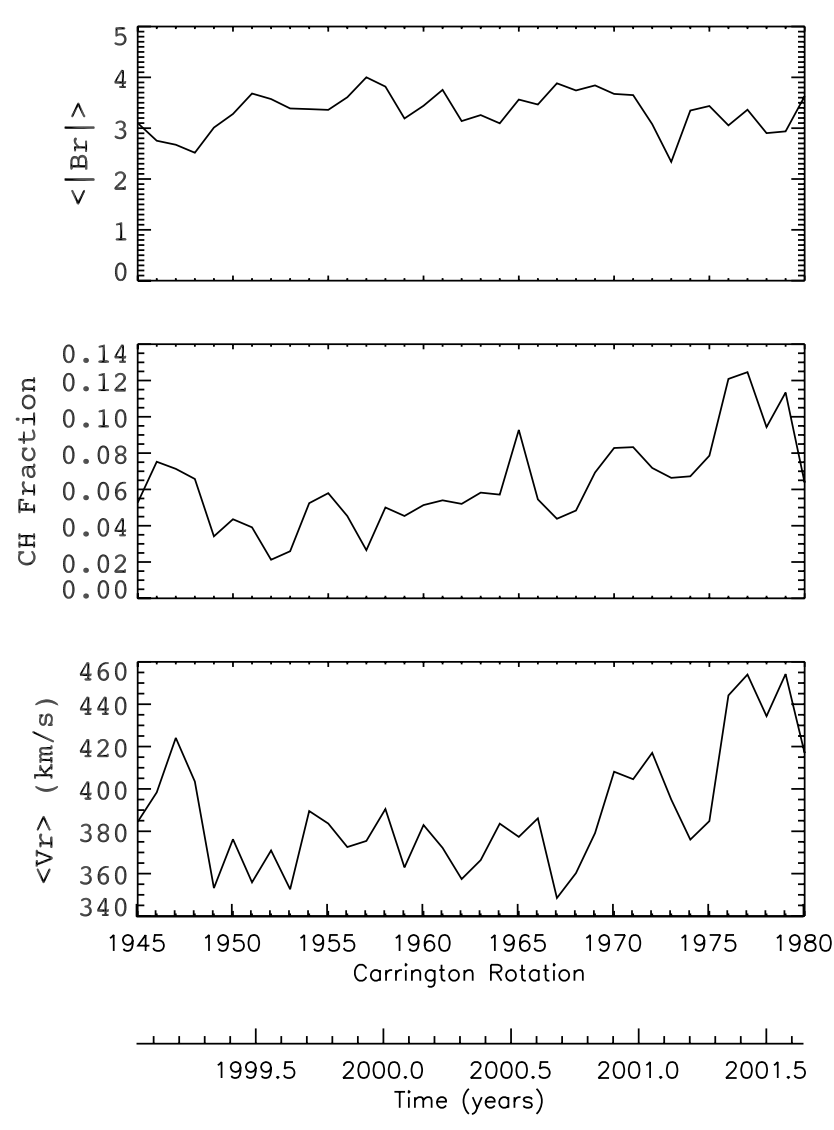

Fig. 5. Evolution of the average magnitude of $B_{r}$ at the photosphere, the fractional area covered by coronal holes, and the average radial speed at $30 R_{S}$ from CR1945 to CR1980.

emental area with increasing distance away from the equator. There is a trend to higher values from CR1952 onward, with the percentage of the solar surface covered by coronal holes rising from $2 \%$ to a maximum of $12.5 \%$. Similarly, in the bottom panel, the average value of the computed radial velocity (itself dependent on the amount of open magnetic field) shows a general increase during later Carrington rotations. The minimum average speed of $\sim 350 \mathrm{~km} \mathrm{~s}^{-1}$ occurred during CR1967 and the maximum average speed of $\sim 455 \mathrm{~km} \mathrm{~s}^{-1}$ occurred during CR1977 and CR1979. It is interesting to note that the time interval corresponding to the lowest average speed (CR1967: 3 September 200030 September 2000) corresponds to the period when Ulysses measured its lowerest speeds (see Fig. 1).

\section{Comparison of heliospheric structure at solar mini- mum and maximum}

Previously we have discussed the evolution of solar wind stream structure during the declining phase of the solar cycle (Riley et al., 2001a). Here, we focus on contrasting the difference between solar minimum and solar maximum conditions. The radial velocity is the primary driver of dynamic evolution, and as we have seen, the main change that occurs 

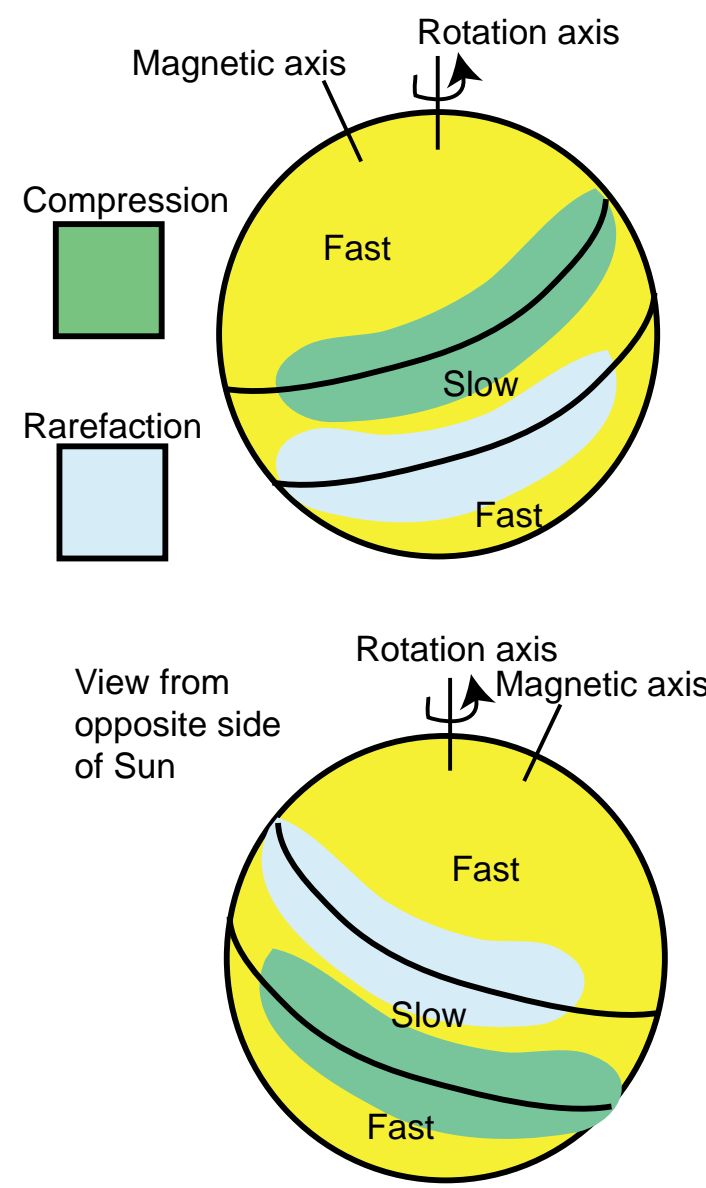

Fig. 6. Schematic of formation and orientation of streams at solar minimum.

during the approach to solar maximum is the loss of a simple band of slow solar wind organized about the heliomagnetic equator, with high-speed wind bounding it on either side at higher latitudes. Near solar maximum, the flow conditions can better be described by slow, and more variable wind essentially everywhere, with pockets of high-speed flow. This fast wind is localized both in latitude and longitude.

Figure 6 is an extension of a schematic presented by Gosling et al. (1993) and illustrates how compression regions and rarefaction regions are generated during the declining phase of the solar cycle. Consider the simple "decliningphase" picture that we have alluded to several times of a band of slow solar wind about the heliomagnetic equator, which is tilted by some amount relative to the rotation axis. A parcel of plasma launched from the northern edge of the slow flow band will eventually be caught by faster plasma to the east (left) of it, as the Sun rotates underneath and replaces the plasma source under that particular radial trace. The net effect is that, far from the Sun, a compression region builds, up organized about this region. When mapped back to the solar surface, the compression region would be located as shown in Fig. 6 (top). On the other hand, in the Southern Hemisphere, a similar argument leads to the formation of a

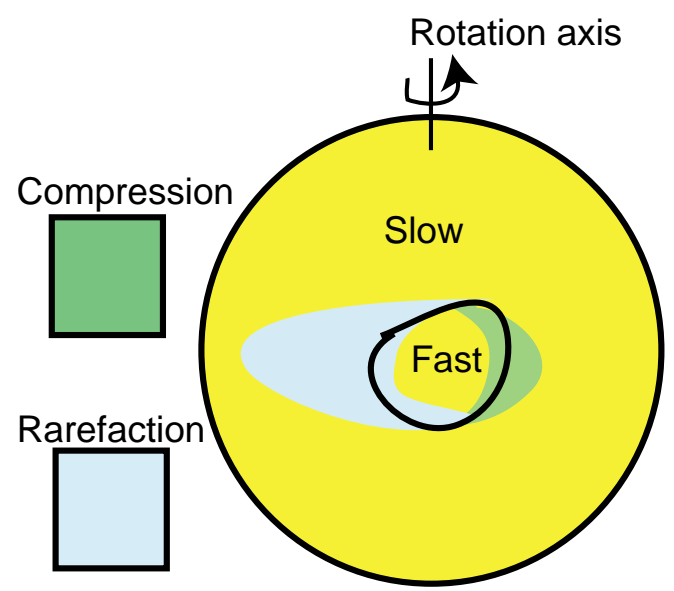

Fig. 7. Schematic of formation and orientation of streams at solar maximum.

rarefaction region, as fast flow now outruns slower flow to the east. Finally, if we look at the opposite side of the Sun, the processes are reversed with a rarefaction region being set up in the Northern Hemisphere and a compression region in the Southern Hemisphere. We can extend the argument to account for more complex shapes of the slow-flow band. In particular, if it is warped or contains more than a single sinusoidal variation with respect to heliographic latitude, the resulting patterns will be richer. Nevertheless, given the flow speed at some inner radial boundary, such as $30 R_{S}$, it is relatively straightforward to infer the resulting dynamical pattern.

At solar maximum, as we have seen, our boundary conditions have much less organization. The simplest case to consider is that of a single equatorial coronal hole giving rise to an isolated high-speed flow that is confined both in latitude and longitude. Such a configuration is summarized in Fig. 7. Applying the same arguments as before, we deduce that slow flow to the west of the equatorial coronal hole is caught by the fast wind, and compressed. East of the coronal hole, the slow flow is outrun by the fast wind generating a rarefaction region. The resulting structures are sideways "V" shapes.

In Fig. 8 we compare simulation results from two extremes of the solar cycle. In the top row we summarize conditions typical of solar minimum (CR1913) at $4.35 \mathrm{AU}$ (corresponding to the heliocentric distance of Ulysses at this time). The panels show solar wind speed, density (logarithmic), and magnetic field magnitude. Note how the slow wind is confined to a band about the equator, with the polar regions consisting entirely of fast wind. For purely radial flow then, compression regions arise where fast flow to the east (left) overtakes slower flow to the west (right). In contrast, rarefaction regions occur when slower to the east (left) is outrun by faster flow to the west (right). Another way to look at this is to consider the Sun rotating underneath a spacecraft that is fixed in an inertial reference frame. The flow pattern rotates such that along a single radial trace plasma is successively launched from points at earlier and earlier longitudes. 


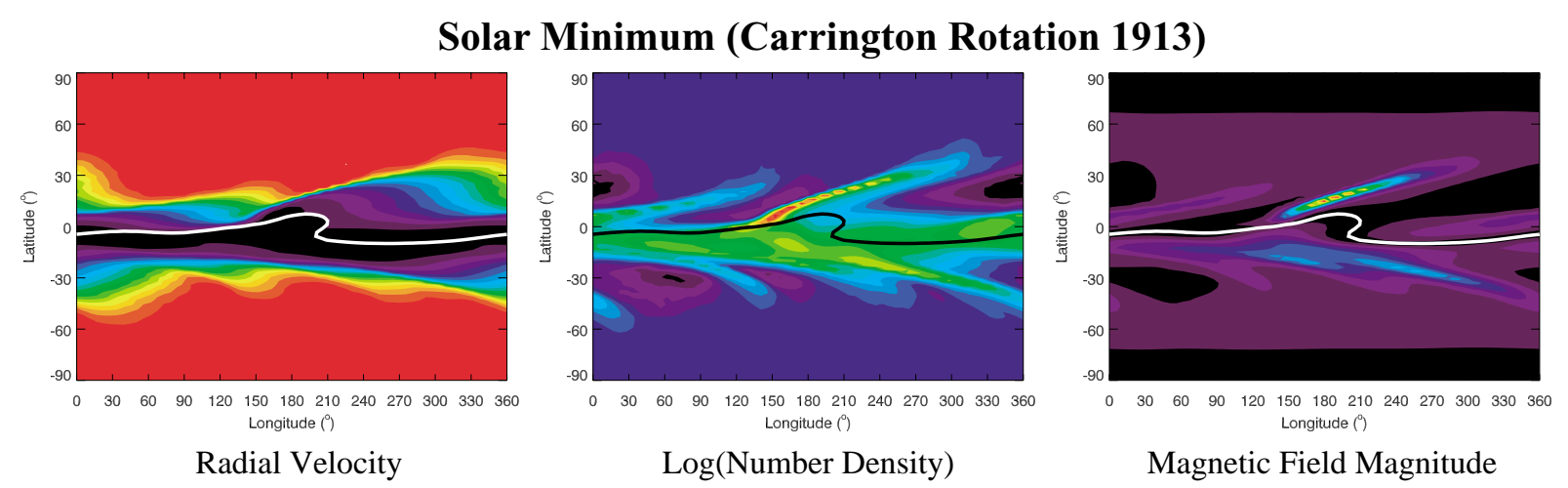

\section{Solar Maximum (Carrington Rotation 1961)}
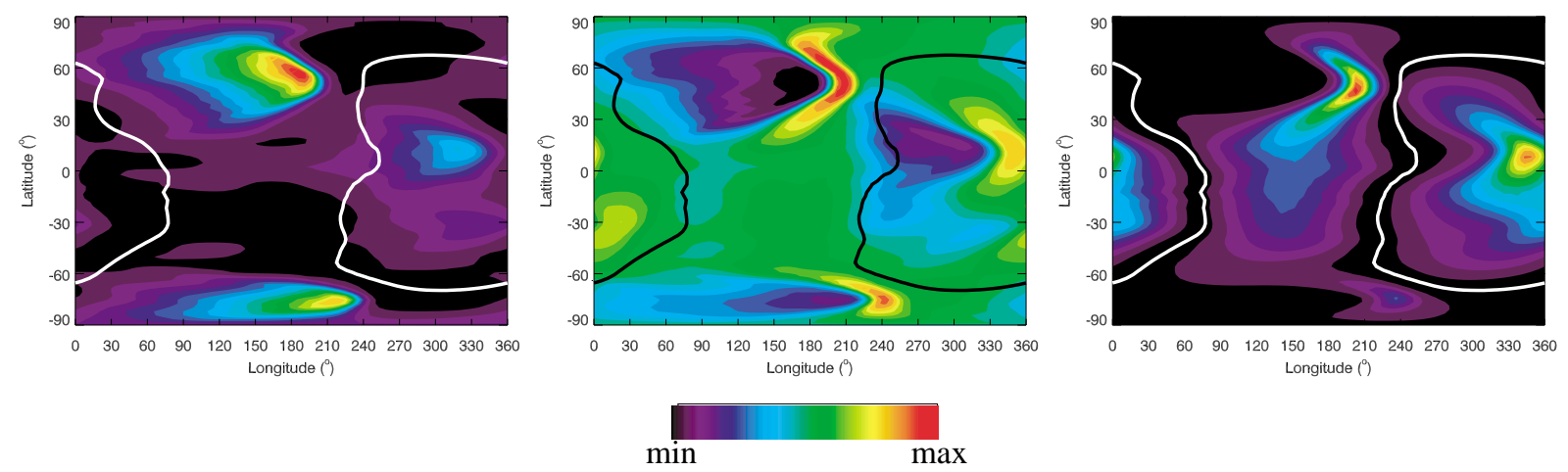

Fig. 8. Comparison of solar wind structure at solar minimum (CR1913) and maximum (CR1961). The heliocentric distance of these slices are 4.35 AU and 4.26 AU, respectively, corresponding to the location of the Ulysses spacecraft at these times. The columns show speed, density, and magnetic field magnitude. The solid white line in each panel denotes the location of the heliospheric current sheet.

A similar argument holds for rarefaction regions. The resultant pattern in the density (and magnetic field strength to a lesser extent) appears as a set of spurs off the slow-flow band.

In contrast, the bottom panels (at 4.26 AU) illustrate conditions at the peak of the solar cycle. Now the picture is one of isolated "beams" of solar wind punching through the slower, more prevalent wind. Where the beam catches slower wind ahead, compression regions form. At the trailing edges of the high-speed streams, rarefactions form. Their limited latitudinal and longitudinal extent, however, now gives rise to "V"-shaped interaction regions. Focusing on the lowermiddle panel, we can identify 3 such profiles. Comparison with the radial velocity, shown in the lower-left panel, indicates that the sources are beams of high-speed wind plowing into slower wind ahead, generating a pressure wave and compressing the plasma. Behind each "V"-compression is a larger expansion wave (or rarefaction region), trailing more than $\sim 180^{\circ}$ in longitude in some cases. Thus, although the high-speed streams are of limited latitudinal and longitudinal extent at the Sun, the rarefaction regions generated by them can span large regions of space.

Superimposed in each panel is the location of the heliospheric current sheet (HCS). The evolution of the HCS over the course of a solar cycle has been discussed by Riley et al. (2002) and the detailed evolution of the interplanetary mag- netic field, together with the HCS, during this time period will be the topic of a future study. Nevertheless, the comparison again serves to highlight the differences between conditions near solar minimum, where essentially all the "action" is confined to a band about the equator, and solar maximum, where the plasma and magnetic structures span essentially all latitudes.

\section{Summary and discussion}

In this report we have used 3-D MHD simulations to model the detailed evolution of the large-scale structure of the inner heliosphere during solar active conditions. In particular, we have used the simulations to describe the global evolution of the inner heliosphere during Ulysses' second orbit descent to the southern polar regions, rapid latitude scan, and arrival into the northern polar regions. We found that the model reproduced the large-scale properties of the solar wind observed by Ulysses, although a detailed analysis of specific intervals is necessary to quantify how well the details of the stream structure are reproduced on the time scale of a single rotation. Preliminary analysis suggests that the model often produces relatively good matches, however, there are often intervals with poor correlation. Whether this is due to approximations in the model, or due to the presence of CMEs 
and other transient phenomena that are not included in the model remains to be seen.

There are several potentially significant sources of error in the model that can lead to inaccuracies in the solution. First and foremost, our resolution is limited to a few degrees in latitude and longitude. While the coronal models employ a non-uniform grid with spacing ranging from $0.018 R_{S}$ to $3.1 R_{S}$, the heliospheric models use a uniform spacing of $15 R_{S}$. As such, our heliospheric solutions cannot resolve shocks, which, because of numerical diffusion, remain steepened waves. Second, our results represent averages over a Carrington rotation. The photospheric magnetic field data used to drive the model was obtained over a period of ' $\sim 26$ days from observations centered on the subsolar point. Thus model results at longitudes significantly different from Earth's position must be carefully considered if significant evolution on the time scale of a solar rotation occurred. Ulysses observations, in particular, suffer from such an affect. Since it remains in essentially an inertial reference frame, it intercepts the Earth's longitude once per year, straying up to $180^{\circ}$ during the intervening times. At worst, then, the measurements made at Ulysses were produced by plasma launched from the opposite side of the Sun from the Earth, and thus, never directly visible from ground-based or nearEarth observatories. Third, we do not account for transient activity, and, in particular, coronal mass ejections. CMEs undoubtedly perturb the ambient solar wind significantly, and we must therefore be careful when interpreting the observations that none were obviously present. Fourth, our method of solution is not completely self-consistent. In particular, we use the expansion of the magnetic field to prescribe the variation in solar wind speed. This has been discussed in more detail by Riley et al. (2001a). And, while reasonable, and borne out empirically (Wang and Sheeley, 1990), it likely does not accurately mimic the more fundamental heating processes that are occurring in reality. Finally, in the absence of any reliable observations, our coronal model specifies uniform temperature and density on the inner radial boundary.

The evolution of the solar magnetic field surrounding solar maximum is complex. At one end of the observational spectrum, we have photospheric observations from which we can infer the radial component of the magnetic field. At the other end, we have in situ observations by spacecraft that provide detailed, although localized measurements of the magnetic field vector. One way to connect the two is through global MHD simulations. Riley et al. (2002) described the evolution of the HCS over the time scale of a solar cycle, including the time period modeled here. They found that during Carrington rotations 1960 and 1961, the typical "ballerina skirt" shape was replaced by a "conch shell"-like shape. In essence, rather than the HCS separating the northern and southern poles, it was localized in longitude, and both poles had the same polarity. This is supported by the photospheric field maps (Fig. 2), which show that during Carrington rotations 1960 and 1961 the polar fields were inward (blue) in both hemispheres. Moreover, when the Ulysses magnetic field polarity measurements were superimposed onto the global HCS pat- tern, the phase of the polarity changes matched well. Without the simulations, the Ulysses observations might have been interpreted as a dipole-like field, as would have been seen during the late-declining phase of the solar cycle. It is also worth noting that this period does not coincide with the inferred magnetic field reversal, which occurred some 10 Carrington rotations later.

The simulation results described in this study are available online at http://sun.saic.com. In addition to the parameters discussed here, we also provide some basic plotting tools to view all plasma and magnetic field parameters. Simulation data are also available, including simple routines to import the data into IDL. A movie showing the evolution of several of the key parameters discussed here can also be downloaded and played on an AVI or Quicktime compatible media player. We plan to expand this database in both directions, covering both the early Ulysses mission, as well as the most recent Carrington rotations. Ultimately, we hope that these simulations will develop into an operational tool, capable of predicting ambient solar wind conditions at Earth (and beyond) with up to 4 days advanced warning.

Acknowledgements. We gratefully acknowledge the support of the National Aeronautics and Space Administration (Sun-Earth Connections Guest Investigator Program, Sun-Earth Connections Theory Program, and Supporting Research and Technology Program) in undertaking this study. We also thank National Science Foundation at the San Diego Supercomputer Center for providing computational support.

Topical Editor R. Forsyth thanks A. Gonzalez-Esparza and G. Erdos for their help in evaluating this paper.

\section{References}

Axford, W. I.: Interaction of the solar wind with the interstellar medium, in: Solar Wind, Space Science Reviews special issue on Outer Solar System Exploration-an Overview, 14, 582, (Eds) Sonett, C. P., Coleman, P. J., and Wilcox, J. M., 1973.

Balogh, A., Beek, T. J., Forsyth, R. J., Hedgecock, P. C., Marquedant, R. J., Smith, E. J., Southward, D. J., and Tsurutani, B. T.: The magnetic field investigation on the ulysses mission: Instrumentation and preliminary scientific results, Astron. Astrophys. Supp. Series, 92, 221-236, 1992.

Bame, S. J., McComas, D. J., Barraclough, B. L., Phillips, J. L., Sofaly, K. J., Chavez, J. C., Goldstein, B. E., and Sakurai, R. K.: The ulysses solar wind plasma experiment, Astron. Astrophys. Supp. Series, 92, 237-265, 1992.

Fisk, L.: Motion of the footpoints of heliospheric magnetic field lines at the sun: Implications for recurrent energetic particle events at high heliographic latitudes, J. Geophys. Res., 101, 15 547-15 554, 1996.

Gonzalez-Esparza, J. and Smith, E.: Three-dimensional nature of interaction regions: Pioneer, voyager, and ulysses solar cycle variations from 1 to $5 \mathrm{AU}$, J. Geophys. Res., 102, 9781-9792, 1997.

Gosling, J. T., Bame, S. J., McComas, D. J., Phillips, J. L., Pizzo, V. J., Goldstein, B. E., and Neugebauer, M.: Lat-itudinal variation of solar wind corotating stream interaction regions: Ulysses, Geophys. Res. Lett., 20, 2789-2792, 1993. 
Gosling, J. T., Feldman, W. C., McComas, D. J., Phillips, J. L., Pizzo, V. J., and Forsyth, R. J.: Ulysses observations of opposed tilts of solar wind corotating interaction regions in the northern and southern solar hemispheres, Geophys. Res. Lett., 22, 3333, 1995.

Gosling, J. T., Bame, S. J., Feldman, W. C., McComas, D. J., Riley, P., Goldstein, B. E., and Neugebauer, M.: The northern edge of the band of solar wind variability: Ulysses at $-4.5 \mathrm{AU}$, Geophys. Res. Lett., 24, 309, 1997.

Levine, R. J., Altschuler, M. D., and Harvey, J. W.: Solar sources of the interplanetary magnetic field and solar wind, J. Geophys. Res., 82, 1061-1065, 1977.

Linker, J. A., Mikić, Z., Bisecker, D. A., Forsyth, R. J., Gibson, S. E., Lazarus, A. J., Lecinski, A., Riley, P., Szabo, A., and Thompson, B. J.: Magnetohydrodynamic modeling of the solar corona during whole sun month, J. Geophys. Res., 104, 98099830, 1999.

McComas, D. J.: The three dimensional structure of the solar wind over the solar cycle, in Solar Wind 10, (Ed) Velli, M., AIP, Pisa, Italy, 2002.

McComas, D. J., Bame, S. J., Barraclough, B. L., Feldman, W. C., Funsten, H. O., Gosling, J. T., Riley, P., Skoug, R., Balogh, A., Forsyth, R. J., Goldstein, B. E., and Neugebauer, M.: Ulysses return to the slow solar wind, Geophys. Res. Lett., 25, 1-1, 1998.

McComas, D. J., Gosling, J. T., and Skoug, R. M.: Ulysses observations of the irregularly structured mid-latitude solar wind during the approach to solar maximum, Geophys. Res. Lett., 27, 24372440, 2000.

McComas, D. J., Elliott, H. A., Gosling, J. T., Reisenfeld, D. B., Skoug, R., Goldstein, B. E., Neugebauer, M., and Balogh, A.: Ulysses second fast-latitude scan: Complexity near solar maximum and the reformation of polar coronal holes, Geophys. Res. Lett., 29, 10.1029/2001GL014 164, 2002a.

McComas, D. J., Elliott, H. A., and von Steiger, R.: Solar wind from high latitude coronal holes at solar maximum, Geophys. Res. Lett., 29, 10.1029/2001GL013 940, 2002b.

Mikić, Z., Linker, J. A., Schnack, D. D., Lionello, R., and Tarditi, A.: Magnetohydrodynamic modeling of the globalsolar corona,
Physics of Plasmas, 6, 2217-2224, 1999.

Pizzo, V. J.: Interplanetary shocks on the large scale: A retrospective on the last decades theoretical efforts, in: Colli-sionless Shocks in the Heliosphere: Reviews of Current Research, Geophys. Monogr. Ser., 35, 51-68, 1985.

Pizzo, V. J.: The evolution of corotating stream fronts near the ecliptic plane in the inner solar system. ii - three-dimensional tilteddipole fronts, J. Geophys. Res., 96, 5405-5420, 1991.

Pizzo, V. J.: Global, quasi-steady dynamics of the distant solar wind 1: Origin of north-south flows in the outer heliosphere, J. Geophys. Res., 99, 4173-4183, 1994.

Pizzo, V. J. and Gosling, J. T.: 3-d simulation of high-latitude interaction regions: Comparison with ulysses results, Geophys. Rese. Lett., 21, 2063-2066, 1994.

Riley, P., Gosling, J. T., Weiss, L. A., and Pizzo, V. J.: The tilts of corotating interaction regions at midheliographic latitudes, J. Geophys. Res., 101, 24 349-24 358, 1996.

Riley, P., Linker, J. A., and Mikić, Z.: An empirically-driven global mhd model of the corona and inner heliosphere., J. Geophys. Res., 106, 15 889-15 902, 2001a.

Riley, P., Linker, J. A., Mikić, Z., and Lionello, R.: Mhd modeling of the solar corona and inner heliosphere: Comparison with observations, in Space Weather, Geophysical Monograph Series, 125, pp. 159-167, American Geophysical Union, Washington, D.C., $2001 b$.

Riley, P., Linker, J. A., and Mikić, Z.: Modeling the heliospheric current sheet: Solar-cycle variations, J. Geophys. Res., 107, 2002.

Sarabhai, V.: Some consequences of nonuniformity of solar wind velocity, J. Geophys. Res., 68, 1555, 1963.

Smith, E. J. and Wolfe, J. H.: Observations of interaction regions and corotating shocks between one and five au: Pioneers 10 and 11, Geophys. Res. Lett., 3, 137-140, 1976.

Wang, Y. M. and Sheeley, N. R., J.: Solar wind speed and coronal flux-tube expansion, Astrophys. J., 355, 726-732, 1990.

Wang, Y. M. and Sheeley, N. R. J.: The high-latitude solar wind near sunspot maximum, Geophys. Res. Lett., 24, 3141, 1997. 